\title{
Spatial-temporal Analysis of Tuberculosis Incidence in Burundi Using GIS
}

\author{
Prosper Masabarakiza ${ }^{1}$, Mahmoud Adel Hassaan ${ }^{2, ~ * ~}$ \\ ${ }^{1}$ Faculty of Health Sciences, University of Martin Lutter King, Bujumbura, Burundi \\ ${ }^{2}$ Institute of Graduate Studies and Research, Alexandria University, Alexandria, Egypt \\ Email address: \\ prosmas02@gmail.com (P. Masabarakiza),mhassaan@hotmail.com (M. A. Hassaan) \\ ${ }^{*}$ Corresponding author
}

\section{To cite this article:}

Prosper Masabarakiza, Mahmoud Adel Hassaan. Spatial-temporal Analysis of Tuberculosis Incidence in Burundi Using GIS. Central African Journal of Public Health. Vol. 5, No. 6, 2019, pp. 280-286. doi: 10.11648/j.cajph.20190506.19

Received: October 8, 2019; Accepted: October 29, 2019; Published: November 5, 2019

\begin{abstract}
Tuberculosis is one of the most contagious diseases that has been present for over 5000 years and it is still one of the most significant public health problems. This paper is intended to employ GIS in analyzing spatial variations of tuberculosis incidence in Burundi highlighting the main hot spots. Also, the paper aims to evaluate the temporal changes of TB incidence during the period 2009-2017 and guide the resource allocation. For this purpose, data on tuberculosis incidence at both province and health district level were analyzed. Data on incidence rate of TB and demographic data were collected at province level. Also, data on cases of TB recorded at health district level were acquired. The collected data were analyzed at both temporal and spatial scale. Temporal analysis involved identifying the various trends of TB incidence rate in various Burundi provinces during the period 2009-2017. Spatial analysis comprised mapping spatial variations in TB incidence rates and their trend over the period 2009-2017 and TB incidence at health district level. Moreover, Hot Spot analysis was performed to delineate those districts of statistically significant hot spots in TB incidence in Burundi. The temporal analysis of TB incidence rate, at province level, revealed that during the period 2009-2017, Burundi provinces have experienced varied trends of TB incidence with an annual change rate ranging between $(-32.9)$ and $(+5.22)$ in case of TB in all clinical forms and between $(-12.2)$ and $(+1.1)$ in case of Pulmonary TB. TB incidence rates were found to be positively correlated with proportion of urban population and population density. Meanwhile, spatial analysis of TB cases, revealed that eastern parts of Burundi have been experiencing relatively low incidence rates of TB compared to other parts of the country. This was highlighted by Hot Spot analysis that revealed a tendency of Pulmonary TB cases to be clustered and a hot spot in Pulmonary TB incidence was clearly distinguished in western parts of Burundi. Spatial temporal analysis highlights the potentials of GIS in recognizing trends and spatial pattern of such a disease and may support designing and implementing control programs and guide the resource allocation.
\end{abstract}

Keywords: Tuberculosis, GIS, Spatial-temporal Analysis, Hot Spot Analysis

\section{Introduction}

Tuberculosis (TB) disease is one of major global public health problems $[1,2]$, with almost 1.6 million deaths in 2017 [3]. TB leads usually to enormous socioeconomic disturbance and hinders nation's development [4]. In this context, positive correlation was noticed between declining TB incidence rate and human development index [5]. To alleviate $\mathrm{TB}$ and its socioeconomic implications extensive budget are allocated for providing health care facilities, network of pathological laboratories, and trained technical manpower [6]. For instance, the total expenditures on TB control programs in all 22 high-burden countries during the period 2010-2012 were estimated to be $\$ 6.37$ billion [7].

Due to its high burden, TB was targeted by sustainable development agenda as one of the main epidemics that should be ended by 2030 [8]. Attaining such a target requires mapping tuberculosis incidence and understanding its spatial pattern. Such mapping and understanding can get insight into main determinants and deriving forces that need to be dealt with. 
Generally, understanding spatial patterns can provide insight into the spatial processes that generated and controlling these patterns [9]. As a prerequisite for understanding spatial pattern should be recognized. Mapping disease incidence, spatial pattern recognition and spatial pattern analysis are of great importance as they assist in delineating areas with high incidence rates of a certain disease at spatial scale. Moreover, at temporal scale, spatial pattern recognition can highlight those areas that have varied change rates of a certain disease [10].

GIS was commonly used in mapping TB for a number of purposes that include, for example, providing an insight into the distribution pattern of TB cases, interpreting TB distribution pattern based on various environmental and socioeconomic determinants, profiling TB transmission pattern, identifying "hotspots" of TB incidence and representing the trends over time. It should be noted that applying GIS in mapping TB incidence requires availability of accurate and updated data at varied spatial scale. In this respect, it was argued that introducing mapping into a health program in many of developing countries can be impeded by lack of accurate and complete data [11].

$\mathrm{TB}$ is one of major public health problems in Burundi, accordingly, Government of Burundi launched TB control program since early 1990s. The program involved providing different facilities in terms of TB diagnosis equipment along with financial support to existing health centers both in public and private sectors including NGOs to provide free $\mathrm{TB}$ test and medication of suspected patients [12]. Nevertheless, TB is still considered as one of the top 10 causes of death in 2016 [13] with mortality rate of 18 cases per 100,000 inhabitants in 2017 [14].

This paper is intended to analyze spatial variations of tuberculosis incidence in Burundi highlighting the main hot spots. Also, the paper aims to interpret the spatial variations in TB incidence rate and evaluate the temporal changes of TB incidence period 2009-2017. This, consequently, may support decision-making and policy making process in terms of planning for intervention and control tuberculosis and reducing disease burden.

\section{Methodology}

Performing temporal and spatial analysis of TB incidence in Burundi, data on incidence rate of Pulmonary TB and TB in all clinical forms were collected at province level during the period 2009-2017 [14]. Up till 2015 Burundi was subdivided into 17 provinces, which comprised 46 health districts. In 2015, the administrative setup was amended through creating a new province; Rumonge province, comprising five health districts that were previously belonging to Bujumbura Rural and Bururi provinces [15]. As data on TB incidence at province level, prior to 2016, was not available for the newly created province; Rumonge province, there was a need to estimate TB incidence in this province. For this purpose, TB incidence for Rumonge province was estimated through calculating the average TB incidence for
Bujumbura Rural and Bururi provinces. Also, data on cases of Pulmonary TB and TB in all clinical forms recorded at health district level during 2016 were acquired [16]. Moreover, demographic data on total population and proportion of urban population at province level were collected [17].

Thereafter, the collected data were analyzed at both temporal and spatial scale. Temporal analysis involved identifying the various trends of TB incidence rate in various Burundi provinces during the period 2009-2017 using MINITAB Ver. 14.1.

Meanwhile, spatial analysis comprised mapping spatial variations in TB incidence rates and their trend over the period 2009-2017 and TB incidence at health district level. Also, the relationships between TB incidence rate and its determinants were investigated. Despite mapping a disease may highlight the spatial variations in such a disease, but it doesn't evaluate the distribution pattern of this disease whether to be clustered, dispersed, or random. So that, the spatial distribution pattern of TB incidence in Burundi at health district level was evaluated through Spatial autocorrelation. Moreover, Hot Spot analysis was performed to delineate those districts of statistically significant hot spots in TB incidence in Burundi.

\section{Results}

During the period 2009-2017, the average incidence rate of TB in all clinical forms ranged between 27 and 456 per 100000 of inhabitants. The highest incidence rate was recorded in in Bujumbura Mairie province. Generally, the eastern provinces in Burundi had recorded relatively low incidence rates of $\mathrm{TB}$ in all clinical forms compared to western and middle provinces (Figure 1a).

Similarly, Bujumbura Mairie province recorded highest average Pulmonary TB incidence rate, which ranged between 19 and 212 per 100000 of inhabitants. Again, it was noticed that eastern parts of Burundi have been experiencing relatively low incidence rates of Pulmonary TB compared to middle and western parts (Figure 1b).

Moreover, temporal analysis indicated that different provinces recorded varied trends of incidence rate of TB during the period 2009-2017. In this respect, it was found that the annual change rates of TB incidence ranged between $(-32.9)$ and $(+5.22)$ in case of TB in all clinical forms and between $(-12.2)$ and $(+1.1)$ in case of Pulmonary TB (Figure 2).

Generally, based on trends of TB incidence, Burundi provinces can be classified into three main groups as follows:

Group I: includes those provinces that recorded a noticeable decreased TB incidence rates. The best example of this category is Bujumbura Mairie province, which despite having highest levels of incidence rates, it has a decreasing incidence rate recording -32.9 in the case of TB in all clinical forms and -12.2 in case of Pulmonary TB.

Group II: involves those provinces that recorded a noticeable increased TB incidence rates. The best examples 
of this category are Kirundo and Bubanza provinces that recorded +5.2 in case of TB in all clinical forms (Figure $3 \mathrm{a}$ )

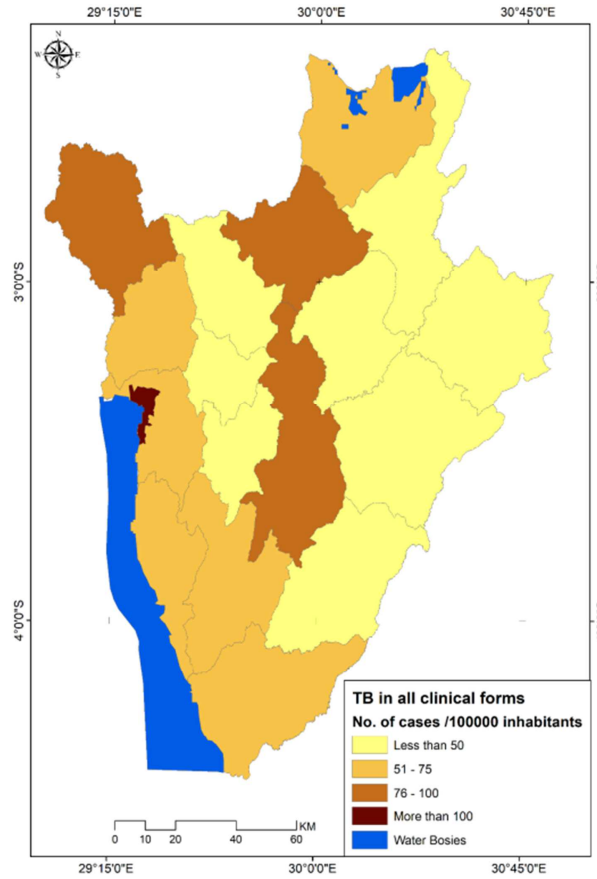

(a) TB in all clinical forms and +1.1 in case of Pulmonary TB (Figure $3 b$ ).

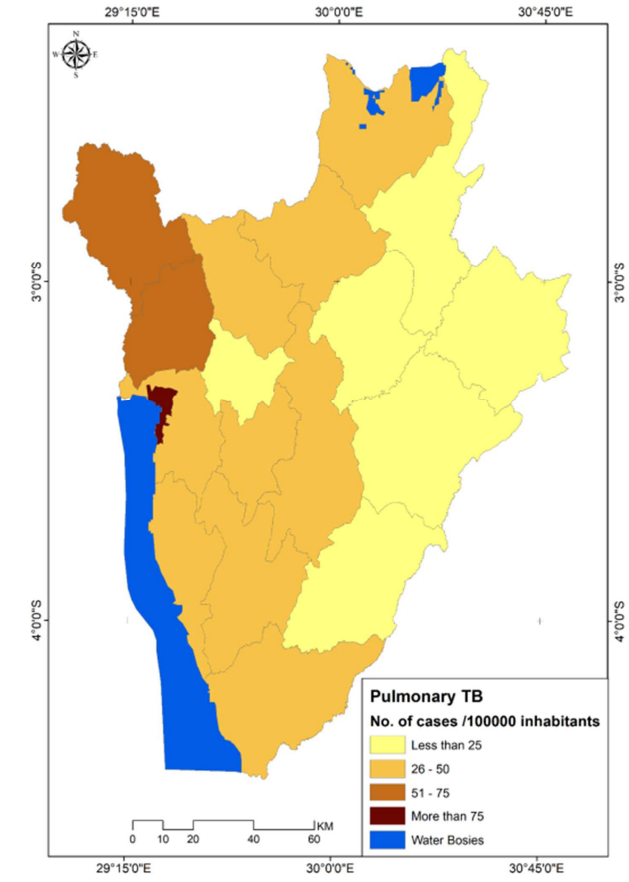

(b) Pulmonary TB

Figure 1. Average incidence rate of TB over the period 2009-2017 in Burundi.
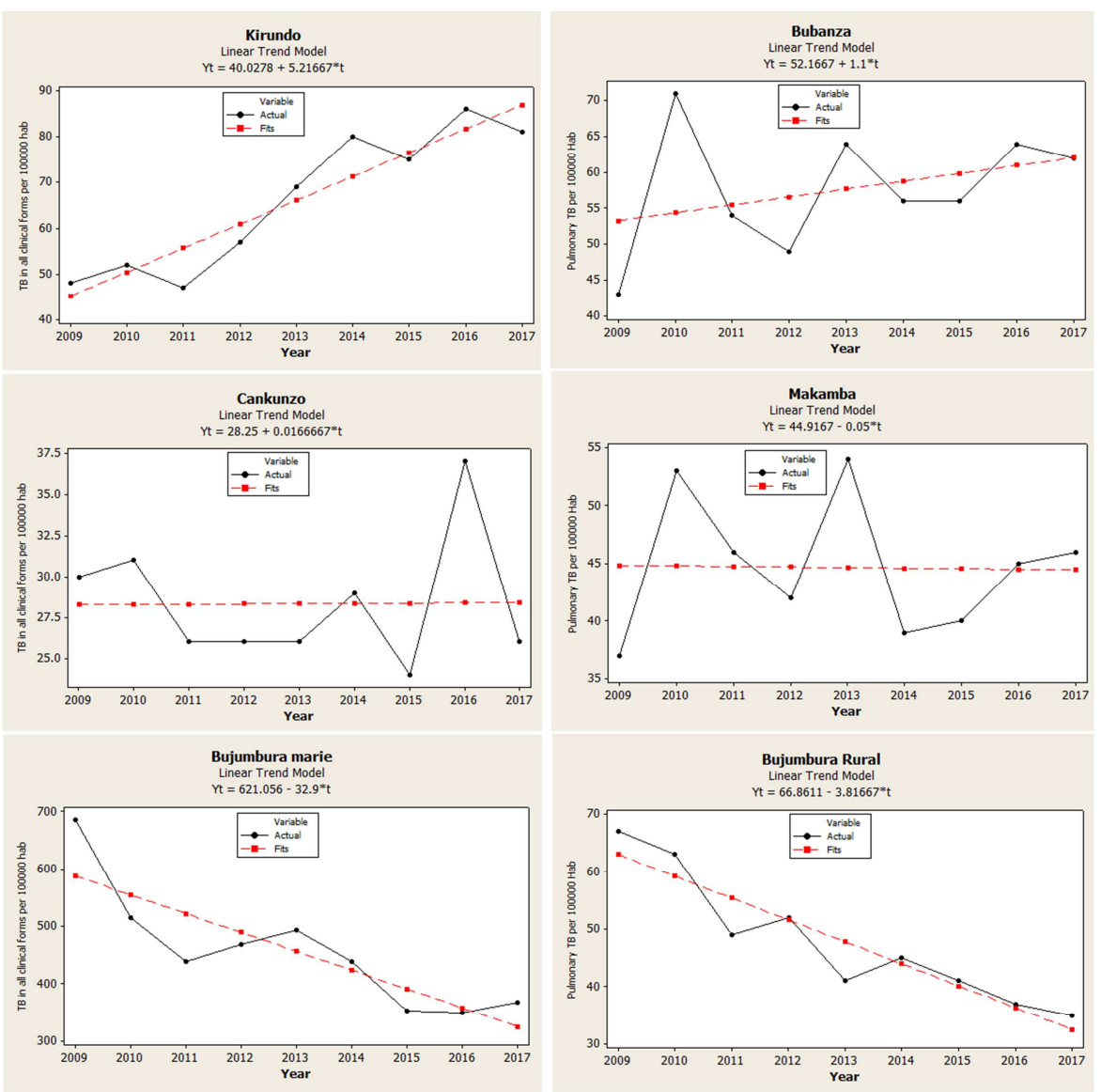

(a) TB in all clinical forms

(b) Pulmonary TB

Figure 2. Trends of TB incidence rates in some provinces during the period 2009-2017. 
Group III: comprises those provinces that recorded insignificant annual change rate of incidence ranging between $(+1)$ and (-1) thus they have a relatively steady

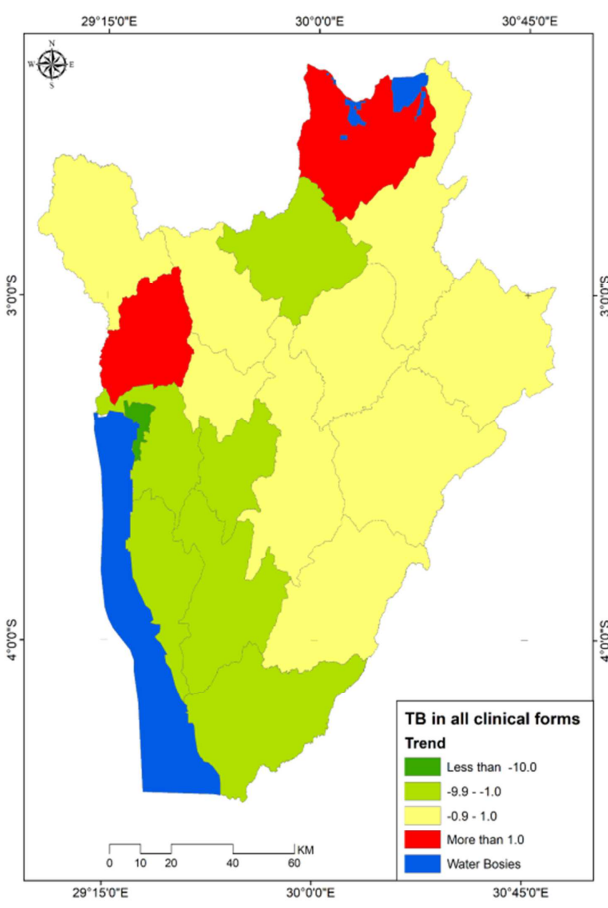

(a) TB in all clinical forms incidence rate. It can be clearly noticed that this category includes major proportions of Burundi.

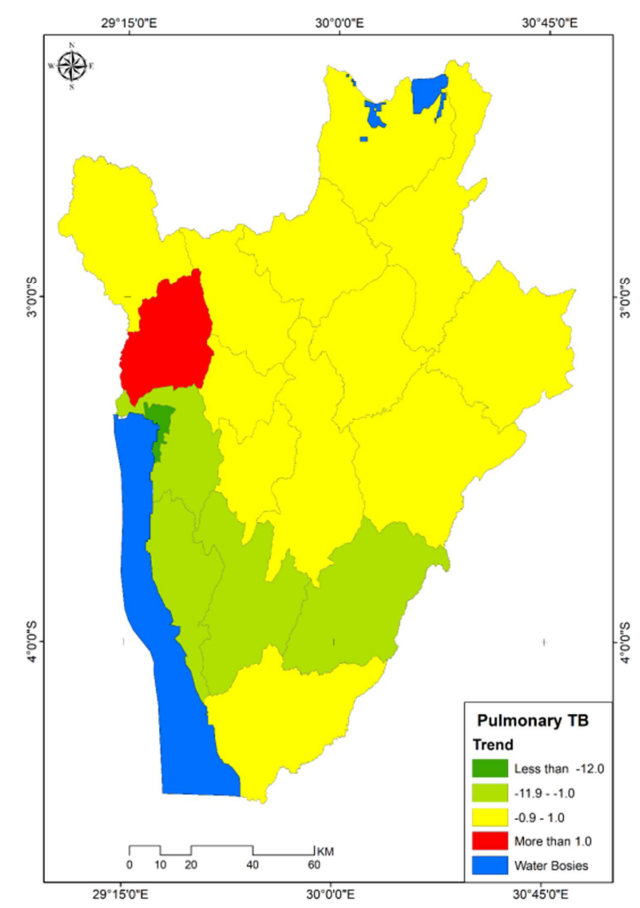

(b) Pulmonary TB

Figure 3. Trends of incidence rates of TB over the period 2009-2017 in Burundi.

At health district level, it was found that high prevalence of TB was found to be located in northwestern parts of Burundi in case of TB in all clinical forms (Figure 4a) and

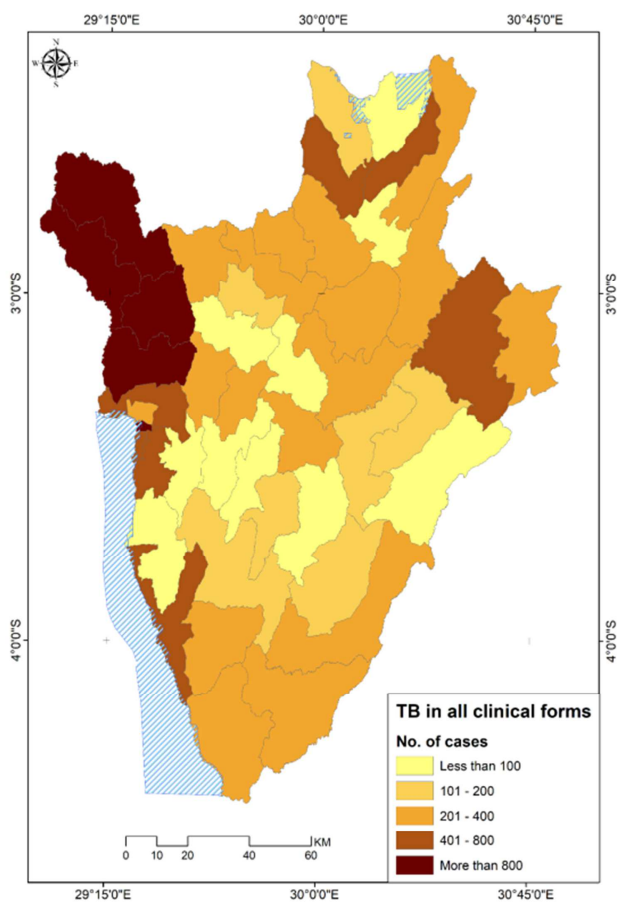

(a) TB in all clinical forms distributed in northwestern, northern and middle parts in case of Pulmonary TB (Figure 4b).

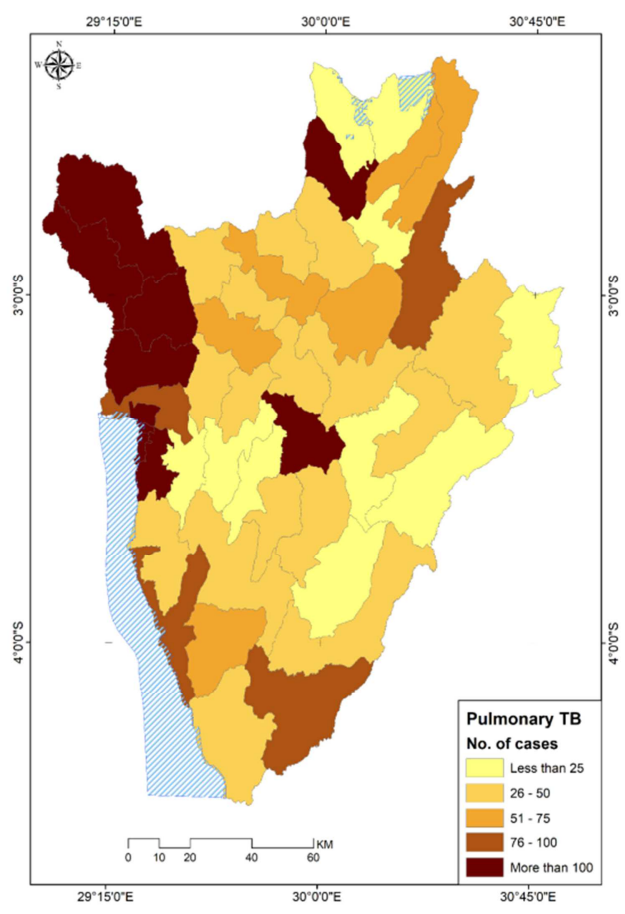

(b) Pulmonary TB

Figure 4. Number of TB cases recorded in Burundi at health district level in 2016. 
To evaluate the distribution pattern of TB incidence rate at health district level, Spatial Autocorrelation analysis (Moran's I index) was performed. In this respect, it was found that the spatial distribution of $\mathrm{Tb}$ cases in all clinical forms is the result of random spatial processes as the $p$-value

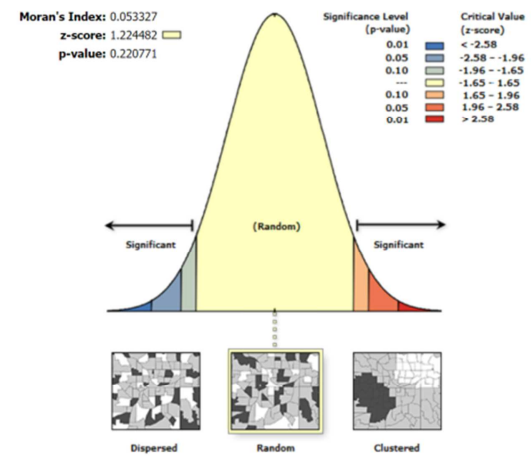

(a) TB in all clinical forms is not statistically significant $(p>0.2)$ (Figure 5a). in contrast, the spatial distribution of high levels of and/or low Pulmonary TB cases is more spatially clustered with Moran's I index for Pulmonary TB cases of $0.79(\mathrm{p}<0.01)$ Figure $5 b)$.

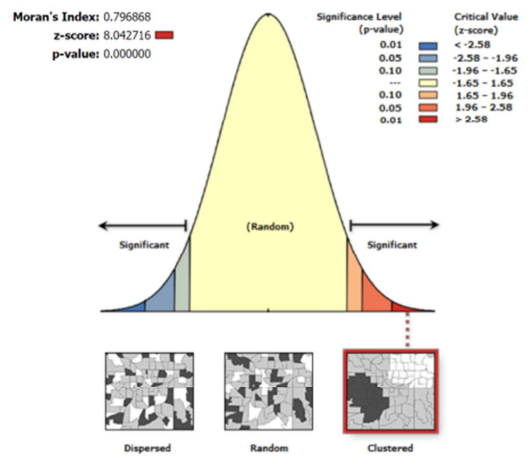

(b) Pulmonary TB

Figure 5. Results of spatial auto correlation analysis.

Thereafter, Hot Spot analysis was performed to identify hot spots of Pulmonary TB cases in Burundi. It was found that hot spots in Pulmonary TB incidence are located in western parts of Burundi, particularly in seven heath districts including: DS Mpanda, DS Isale, DS Bujumbura Nord, DS
Bujumbura Centre, DS Bujumbura SUD, DS Kabezi and DS Rwibaga (Figure 6). This highlights the main health districts with highest $\mathrm{TB}$ incidence and thus need to be targeted by intervention programs.

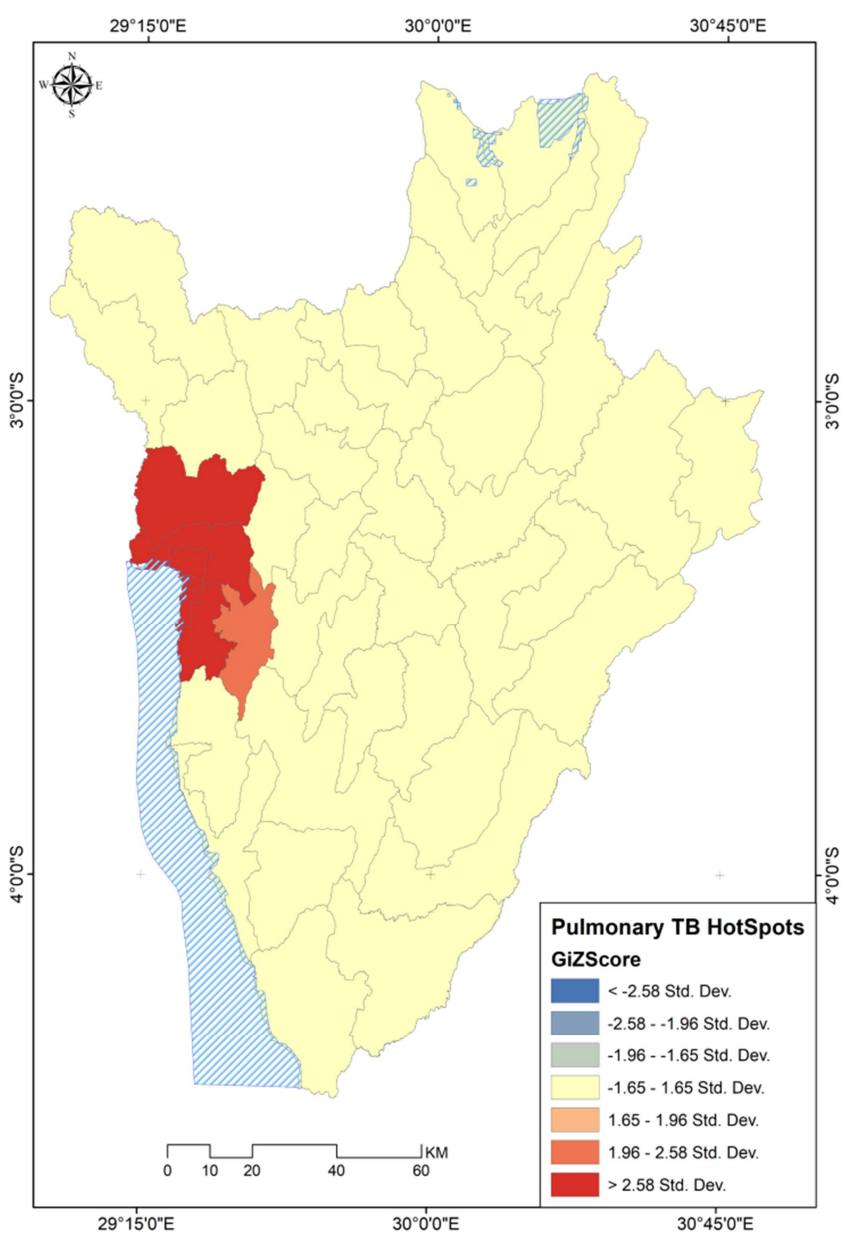

Figure 6. Hot Spot of Pulmonary TB incidence in Burundi. 


\section{Discussion}

It should be noted that the seven health districts with highest TB incidence rate, host together about $14 \%$ of the total population of Burundi in 2015 and have $32 \%$ of the total number of hospitals and about $22 \%$ of the total number of health facilities in the country [16]. This, may shed the light on the quality of provided health services in these districts and efficiency of intervention programs.

Usually, average TB incidence rates during the period 2009-2017 were found to be high in urban areas with high population density and crowded living conditions. Thus, an association between increased TB incidence rate and rapid urbanization was suggested [18]. To examine such an association, correlation coefficients between TB incidence rate on one hand and the proportion of urban population and population density, at province level, on the other hand were calculated. Generally, Pulmonary TB incidence rate was found to be positively correlated with proportion of urban population and population density with correlation coefficients of +0.955 and +0.940 , respectively ( $\mathrm{P}$ value $<$ 0.01). Similarly, there was a strong positive correlation between incidence rate of TB in all clinical forms and the two variables with correlation coefficients of +0.987 and +0.977 , respectively ( $\mathrm{P}$ value $<0.01)$.

To investigate the relationship between proportion of urban population and population density as independent variables and average $\mathrm{TB}$ incidence rate as a dependent variable, a regression analysis was performed. The results showed a significant relationship between proportion of urban population and TB incidence rates $(\mathrm{p}$ value $<0.01)$ with adjusted R2 value of 0.907 and 0.973 in case of Pulmonary TB and TB in all clinical forms, respectively. This means that $90.7 \%$ and $97.3 \%$ of the variation in Incidence rate of Pulmonary $\mathrm{TB}$ and $\mathrm{TB}$ in all clinical forms, respectively, can be explained by the variations in proportion of urban population. Also, regression analysis emphasized that TB incidence rates were found to be significantly related to population density ( $\mathrm{p}$ value $<0.01$ ) with adjusted R2 value of 0.877 and 0.951 in the case of Pulmonary TB and TB in all clinical forms, respectively. This emphasized the role of population density and urbanization in TB incidence in Burundi. Therefore, any invention and control program should target urban centers with high population densities.

\section{Conclusion}

Using GIS in performing temporal and spatial analysis of tuberculosis incidence can play a crucial role in recognizing trends and spatial pattern of such a disease. This, in turn, may considerably support designing and implementing control programs and guide the resource allocation. The application of GIS in performing spatial and temporal analysis of TB requires availability of data on $\mathrm{TB}$ cases and incidence rates at different levels.
The trend analysis of TB incidence rate in Burundi at province level during the period 2009-2017 showed that major proportions of Burundi provinces have been experiencing a relatively steady incidence rate.

Spatial analysis indicated TB cases, particularly, Pulmonary TB, tend to be clustered in the western parts of Burundi, where main hot spots were identified. The distinguished hot spot including seven health districts, where a considerable proportion of total hospitals and facilities are located. This may raise a question about the effectiveness of current TB control program in Burundi.

A significant relationship was noted between TB incidence rate, on one hand, and proportion of urban population and population density, on the other. This emphasized the role of both factors in tuberculosis incidence in Burundi. This means that future programs for reducing tuberculosis burden in Burundi should primarily target urban areas and heavily populated areas.

\section{References}

[1] Ahmed, A., et al., Incidence and determinants of tuberculosis infection among adult patients with HIV attending HIV care in north-east Ethiopia: a retrospective cohort study. BMJ Open, 2018. 8: e016961.

[2] Harling, G., et al., Determinants of tuberculosis transmission and treatment abandonment in Fortaleza, Brazil. BMC Public Health, 2017. 17 (1): p. 508.

[3] WHO, W. H. O., Global tuberculosis report 2018. 2018: Geneva. p. 277.

[4] Ananthakrishnan, R., et al., Expenditure Pattern for TB Treatment among Patients Registered in an Urban Government DOTS Program in Chennai City, South India. Tuberc Res Treat, 2012. 2012: p. 747924.

[5] Dye, C., et al., Trends in tuberculosis incidence and their determinants in 134 countries. Bulletin of the World Health Organization, 2009. 87 (9): p. 683-691.

[6] Ravichandran, N., Tuberculosis Control in developing countries: A Generalized Community Health Worker Based Model. 2004, Indian Institute of Management: Ahmedabad.

[7] Macintyre, K. and B. Mwangi, Expenditure reported by national Tuberculosis programs in 22 high burden countries between 2010 - 2012: what is the Global Fund's contribution? 2014, Aidspan: Nairobi.

[8] UN, U. N., Sustainable Development Goals. 2015, United Nations.

[9] Williams, E. A. and E. A. Wentz, Pattern Analysis Based on Type, Orientation, Size, and Shape. Geographical Analysis, 2008. 40 (2): p. 97-122.

[10] Jacquez, G., D. Greiling, and A. Kaufmann, Spatial pattern recognition in the environmental and health sciences: a perspective, in GEOIDE Workshop. 2001, Terra Seer: Quebec City, Canada $\mathrm{p}$. 
[11] Partilla, M. The uses of mapping in improving management and outcomes of tuberculosis control programs: an overview of available tools. 2008 [cited 201817 October]; 18]. Available from:https://www.challengetb.org/publications/tools/hss/Uses_of Mapping_Improving_Management_Outcomes_of_TB_Control_ Programs.pdf.

[12] MSPLS, M. d. 1. S. P. e. d. 1. 1. c. 1. S., Plan stratégique de lutte contre la tuberculose 2011-2015. 2010: Bujumbura.

[13] IHME, I. f. H. M. a. E. Burundi. 2018 [cited 20185 November]; Available

from: http://www.healthdata.org/burundi.

[14] MSPLS, M. d. 1. S. P. e. d. 1. 1. c. 1. S., Programme National lèpre Tuberculose Rapport Annuel 2017. 2017: Bujumbura.
[15] Moise, I. K., et al., Seasonal and Geographic Variation of Pediatric Malaria in Burundi: 2011 to 2012. Int J Environ Res Public Health, 2016. 13 (4): p. 425.

[16] MSPLS, M. d. 1. S. P. e. d. 1. 1. c. 1. S., ANNUAIRE STATISTIQUE SANITAIRE 2016. 2016: Bujumbura.

[17] City Population. Burundi: Provinces, major cities \& urban localities 2019 [cited 2019 \& June]; Available from: https://www.citypopulation.de/Burundi-Cities.html.

[18] Lonnroth, K., et al., Drivers of tuberculosis epidemics: the role of risk factors and social determinants. Soc Sci Med, 2009. 68 (12): p. 2240-6. 